\title{
AtrR, an essential regulator of azole resistance in Aspergillus fumigatus, acts via the TR34 element in the cyp51A promoter
}

\author{
W. Scott Moye-Rowley, Department of Molecular Physiology and Biophysics, Carver \\ College of Medicine, University of lowa, lowa City, IA 52246, USA.
}

Introduction: Previous work identified AtrR as an important regulator of azole resistance in $A$. fumigatus via its transcriptional control of an $A B C$ transporter gene and the cyp51A locus encoding the azole target enzyme. Here we identify the binding site for AtrR called the AtrR Response Element (ATRE) with a focus on the TR34 element found in the cyp51A promoter.

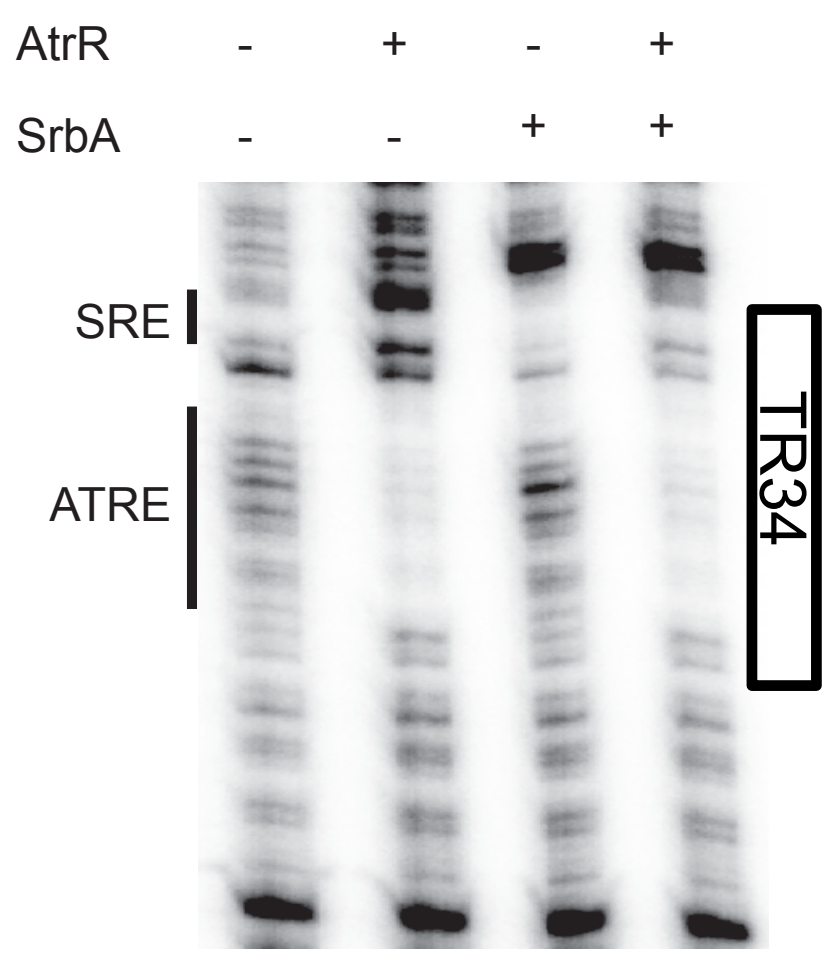

A.

B.

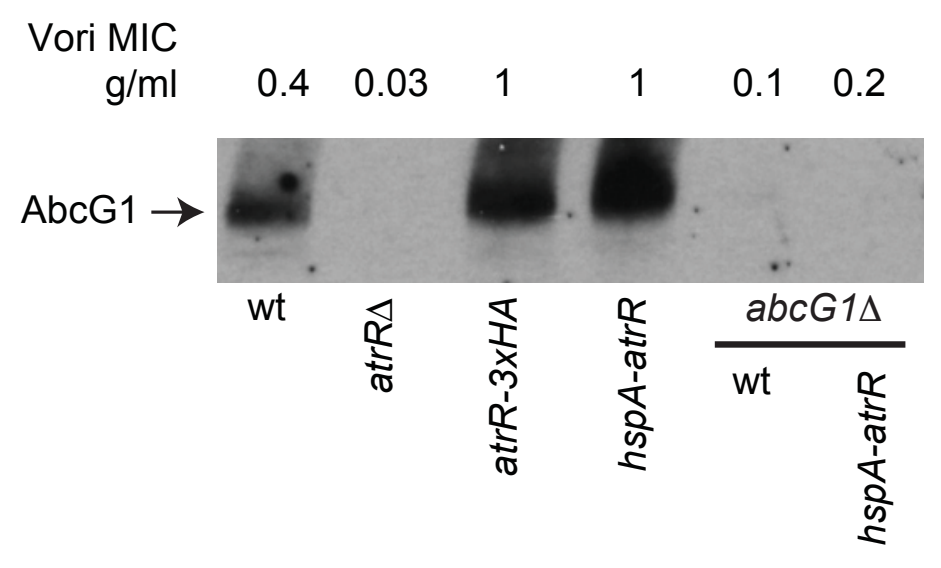

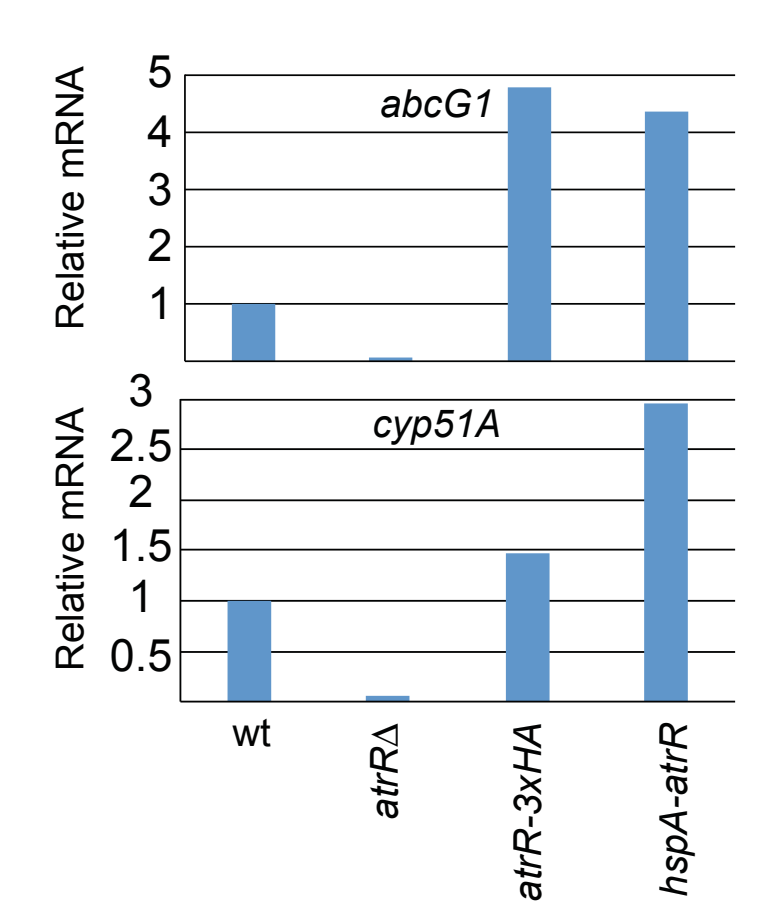

Figure 2. Mutant forms of AtrR are hyperactive. A. Western blot of AbcG1 $A B C$ transporter expression in the indicated strains is shown along with the MIC for voriconazole (Numbers above each lane). The two hyperactive alleles of atrR correspond to either an epitope-tagged allele (atrR-3X HA) or an overproduced but untagged form ( $h s p A-a t r R)$. B. Levels of the indicated mRNAs were determined by RT-qPCR.

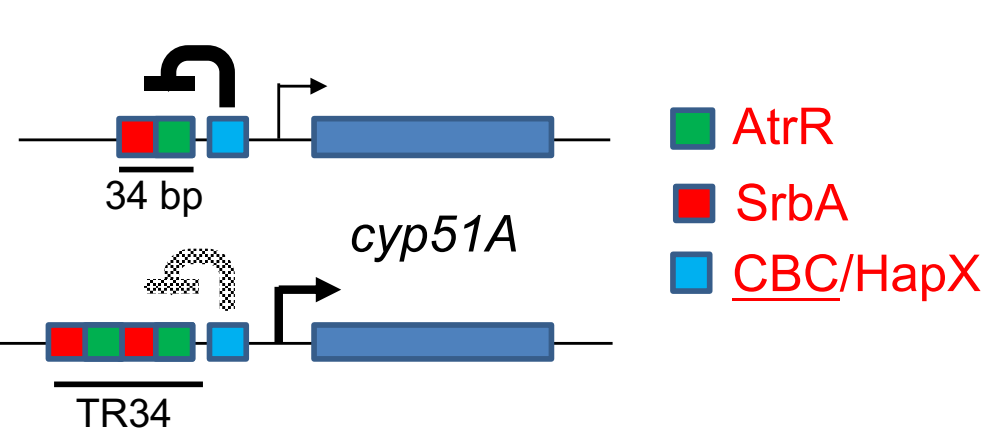

B. $\begin{array}{cc}\text { GAATCACGCGGTCCGGATGTGTGCTGAGCCGAATGAATCACGCGGTCCGATGTGTGCTGAGCCGAATGAAAGTTGCCTAATTACTAAGG } \\ \text { TR34 repeat } & 34 \mathrm{bp}(\mathrm{wt})\end{array}$

Figure 4. Model for transcriptional regulation of cyp51A. A. The location of the ATRE and SRE are indicate as colored boxes. The repression via the CBC/HapX complex (Gsaller, et al PLOS Pathogens 14:12, 2016) is indicated by the solid T bar. The presence of the TR34 duplication is postulated to weaken this repression leading to elevated cyp51A transcription. SrbA response element (SRE) and ATRE are indicated at the right. B. DNA sequence of the elements in $A$.

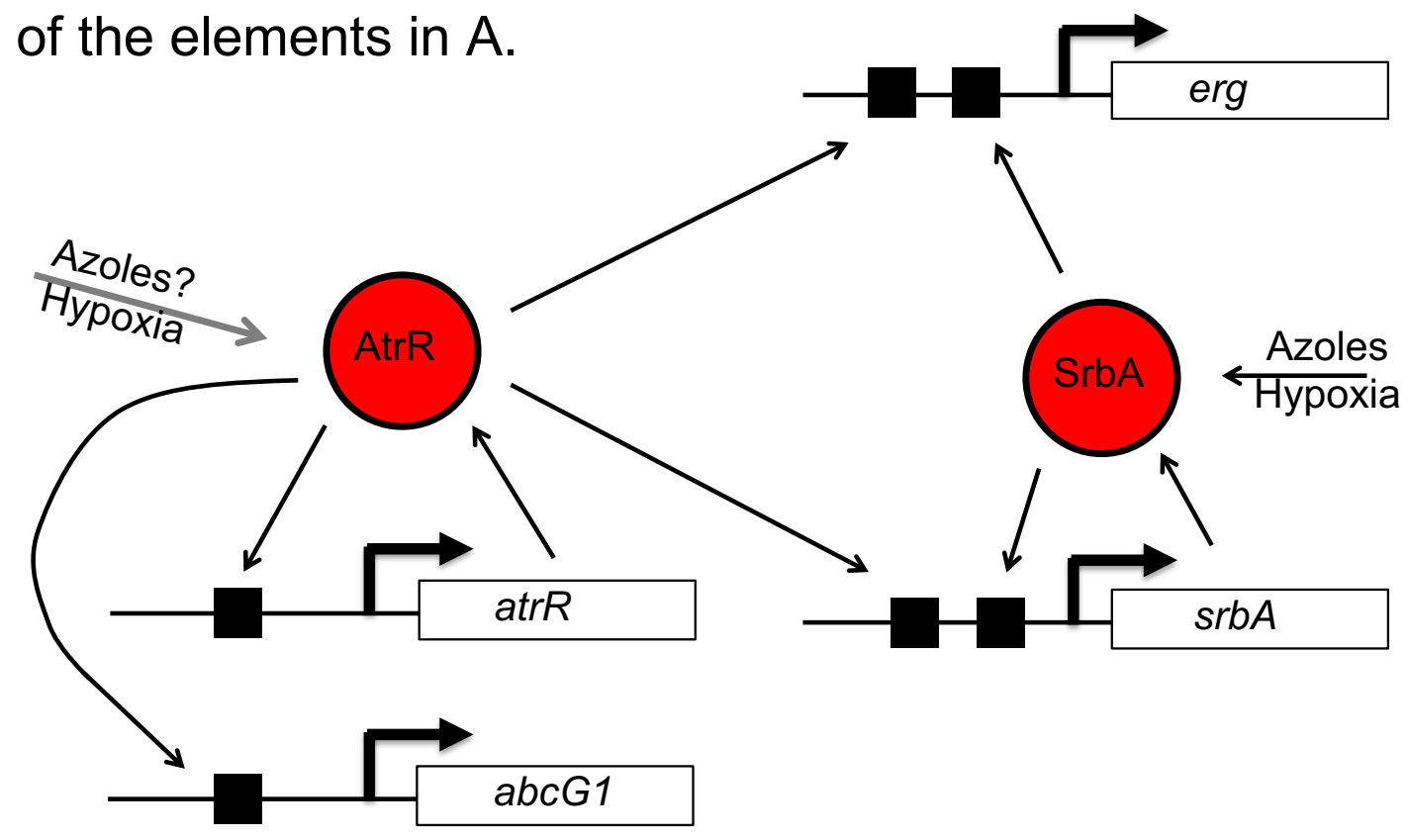

Figure 5. Genetic relationships between AtrR, SrbA and selected target genes. Both AtrR and SrbA control expression of ergosterol pathway genes (erg) but only AtrR regulates transcription of the $\mathrm{ABC}$ transporter-encoding gene abcG1.

A
Figure 3. Epistatic relationship between atrR and srbA. The indicated strains were plated on minimal media and a disk containing $1 \mathrm{mg} / \mathrm{ml}$ voriconazole placed in the center. 